\title{
Design of H265 Stream Real - time Transmission System Based on Hi3516A
}

\author{
Changqing $\mathrm{Du}^{1, \mathrm{a}}$ \\ ${ }^{1}$ School of Information Engineering, QuJing Normal University, QuJing, Shandong, China, 655000
}

Keywords: H265, Stream Real-Time Transmission System, Hi3516A

\begin{abstract}
With the development of society, video surveillance technology has gone into the era of network monitoring. High-definition, high frame rate and high compression rate have become the new development goals. In this context, the current popular H.264 compression coding standard exposes the limitations such as great storage space requirements and higher network bandwidth occupies and the H.265 is the latest compression coding standard to solve such problems. Therefore, we need to design the H.235 compression coding standard with Hi3516A processor and a real-time streaming media transmission system is designed to promote the development of video surveillance technology.
\end{abstract}

\section{Introduction}

With the rapid development of China's economic strength, the scale of Chinese cities has been expanding and the construction of urban security facilities has become more and more important and urgent. Since 2009, with the financial transportation and other industries and the safety of urban projects on the high demand for high-definition monitoring, In addition to the Shanghai World Expo Guangzhou Asian Games and the Shenzhen University and other large-scale activities to promote the high-definition network video surveillance related products such as mushrooming rapid growth in recent years, whether it is an emergency disaster or city police security incidents are citizens of life and property Impact, therefore, security industry emerged more demand, especially the video surveillance industry, accompanied by network and digital tide, the emergence of a lot of new technology.

With the construction of the first batch of safe cities to today, the video surveillance system has undergone several changes in conversion, has been fully into the digital network of high-definition intelligent stage of the traditional front-end analog camera and network video encoding device is destined to be phase out of this one of the main factors are front-end camera clarity limitations, but also the back-end compression device compression resolution is difficult to continue in the original program has been greatly improved, many end users have been looking for a clearer and more intelligent More simple video surveillance system solutions So some have strong research and development strength of the video surveillance manufacturers began to follow the needs of users continue to explore the summary, and constantly developed a series of high-definition audio and video processing technology of this high-definition digital network Audio and video technology began to appear in the field of video surveillance on the stage, they bring the visual impact in the previous video surveillance system is unimaginable city video surveillance system as a complex system, the ultimate goal is High-definition network of intelligent, full HD video surveillance system is quietly changing the traditional safe city video surveillance system structure model.

\section{The Research on H.265 Core Technology}

The macro-block size is extended to $64 * 64$ and is encoded by the encoding unit, the prediction unit and the transforming unit to improve the coding efficiency. The coding unit is equivalent to the macro-block in H.264, and the prediction unit is used in the coding process. The basic unit of the operation and the transformation unit is the basic unit of the transformation and quantization operation. Will be part of the separation, so that all aspects of processing more flexible.

Using an adaptive transformation technique, that is, the residual four cross tree transformation 
structure. This technique is an extension of H.264 adaptive transform technology. In the process of coding, the size of the correlation transform block can be adjusted adaptively according to the characteristics of motion compensation block size and intra prediction residual. After this, a better energy concentration effect can be achieved, and more image detail can be saved after quantization, but it is also necessary to adjust the size of the transform block according to the current signal characteristics to prevent the ringing effect.

In the encoder loop, the sampling point adaptive offset and adaptive loop filtering technology are realized. Adaptive bias filtering reduces the distortion by adding and subtracting an offset to each type of image. The adaptive loop filter achieves the purpose of reducing the variance value of the reconstructed image and the original image by restoring the reconstructed image.

The parallel operation is introduced, which includes the technology of brick structure division, entropy slice division and wave front parallel processing. The structure of the brick structure divides the image into multiple rectangular regions, and the regions can be processed independently to realize the parallel work. The entropy slicing technique divides the entropy slices and independently decodes the multiple sub-entropy slices to realize the parallel codec Wave front parallel processing technology requires that the coding parameters of the original line be initialized so that the coding process of the previous line is coded and the parallel processing capability of the codec can be improved.

\section{The Network Video Transmission Technology}

Network video transmission refers to the target side of the video data through the way to the user terminal to send, usually between the server and the client, the two sides need to follow the specific communication protocol, such as TCP, UDP, RTP, RTCP, RTSP protocol. The network video transmission is divided into sequential transmission and real-time streaming according to the function. The sequential transmission is realized through the HTTP protocol and the Web server, and the real-time streaming is realized by RTCP / RTP / RTSP. This topic requires the real-time video images taken by the camera to be transmitted to the client, and need to support the RTSP protocol, so real-time streaming mode to achieve. In the real-time streaming media transmission protocol, RTP protocol is responsible for voice, image, fax and other multimedia data packet and real-time transmission; RTCP protocol RTP packet transmission process of transmission quality QoS monitoring and feedback, so as to ensure the quality of data transmission; And RTSP protocol is responsible for streaming media transmission control services. Common real-time streaming media transmission methods are two: based on JRTPLIB library and based on Live555 open source code.

In the first scenario, the JRTPLIB library encapsulates the network protocol such as RTCP / RTP / RTSP. The user calls the API to implement the protocol and completes the specific function. However, JRTPLIB does not send some real-time data. The user needs to customize the RTP Subcontracting and packaging, flow control, congestion control and rate control logic, the design is more difficult.

The second option, Live555 is a C ++ language written streaming media open source project, the use of open standard protocol SIP, RTP / RTCP, RTSP, support H265, H264, MPEG and a variety of audio encoding and other formats, and cross- Run; However, Live555 design is the original intention of the file streaming media services, the framework is only for the file flow, in order to support real-time streaming multimedia devices, the need for specific multimedia equipment to do a greater modification and optimization.

\section{The Application of the Streaming Media Server in the HI3516}

Establish RTSP Server Based on Live555. Live555 is a cross-platform C ++ open source project that provides a solution for streaming media. It enables support for standard streaming media protocols such as RTP / RTCP RTSP SIP. Live555 enables audio and video data for a variety of audio and video encoding formats Streaming support and processing support, including MPEG 
H.264 DV JPEG video and a variety of audio coding At the same time due to good design, Live555 is very easy to expand support for other formats At present, Live555 has been used for a variety of players stream Media playback function, such as VLC. In order to achieve the purpose of real-time video transmission

Live555 Streaming Media Framework. From the structure of the program, the live project includes four basic library program entry classes (in media Server) and some test code (in test Progs). The four base libraries are Usage Environment Basic Usage Environment group sock and live Media. Create process:

1. Create Task Scheduler: here only initializes an fdset and the number of sockets is initialized to 0

2. Create a Usage Environment object with Task Scheduler as a parameter

3. Create the RTSP Server object with the environment and service port number (554/8554) and the user authentication object as parameters. Here is the creation function of the subroutine Dynamic RTSP Server. Create the socket in the create New member function, assign the send buffer, and create the RTSP Server Object here through the turn On Back ground Read Handling function to handle the handle and handle the function associated

4. Execute env-> task Scheduler (). Do Event Loop ();

5. RTSP Server (RTSP Server), in the client to listen to the socket request, will create a RTSP Client Session class and the client for RTSP session, including "OPTIONS, DESCRIBE, SETUP, TEARDOWN, PLAY, PAUSE, GET_PARAMETER, SET_PARAMETER" and The server Media Session is created in DESCRIBE to provide media services

6.live555 through this real-time transmission H264 bit stream to read the source code, you can see H264VideoFileServerMediaSubsession, sink using H264VideoRTPSink, source using H264VideoStreamFramer, the two nodes to insert a lot of other nodes: Byte Stream File Source -> H264VideoStreamParser-- > H264VideoStreamFramer -> H264FUAFragmenter -> H264VideoRTPSink.

The Cutting and Transplantation of Live555 in the Hi3516. This article is based on the above principle, after cutting, by collecting images and h264 encoding, access to a h264 source, and then use it instead of Byte Stream File Source, the source called H264ByteStreamSource. In order to improve efficiency, acquisition and coding part has been placed in another thread It can be seen, parser () is its own buffer, and its size is fixed: define BANK_SIZE 150000 However, we write Source, each output is a frame of data, including multiple NALU, as long as a Frame does not exceed 150000 bytes, you can complete the reliable transmission of video.

\section{The Application of WEB Server in the HI3516}

With the development and popularization of Internet technology, people's lives more and more closely linked with the network embedded technology has been widely used in various fields, more and more information products need to access Internet users will be able to Web Page for remote access and control to view and modify the information about the remote device of interest in the embedded device to join the Web Server, you can provide users with Web-based graphical management interface, the user quickly and easily use the phone or PC browser Access to embedded devices for parameter viewing and setting and various operations, this will not only make the ease of use of embedded devices greatly improved, and convenient unified management

Currently popular web server httpd boa thttpd shttpd lhttpd, etc., in the embedded use of the most widely is the main boa httpd and thttpd HTTPD is the simplest of a Web Server, its function is the weakest, does not support authentication, does not support CGI THTTPD And BOA support certification CGI, etc., are more full-featured BOA is a single task of small HTTP server, the source code open performance is excellent, especially suitable for use in embedded systems.

The Design Principles of Web Server. Embedded web server based on the TCP / IP protocol stack, the need to achieve HTTP, TCP and UDP protocols Any one of the clients, through the HTTP protocol and embedded Web Server to establish a connection Embedded Web server in the Web browser and devices Provides a unified GUI interface, so that the client can be as transparent as the 
local monitoring of the status of management equipment.

The Principle and Application of CGI. CGI (Common Gateway Interface) is the technology used to build dynamic web pages. When a client requests a CGI program specified on a Web server, the Web server starts a new process to execute certain CGI programs, After the implementation of the results of the results in the form of web pages back to the client CGI advantage is that it can be written in many languages, such as $\mathrm{C}++\mathrm{VB}$ and Perl language in the language of choice has great flexibility The most commonly used CGI development language Perl CGI describes a standard for transferring data between a client and a server program.

CGI is generally a separate executable program, in essence, CGI is a Web server-side process, whenever the client input a request, you must activate a CGI program can be used as a Web server and other application information resources and database between Of the intermediary through the CGI interface standard, the Web server can call a CGI program, while the user-specified data passed to it, then the CGI program according to the incoming data to do the corresponding treatment, which of course can also be a database access Processing, and finally the Web server and then return the results of the program to the Web browser.

\section{The Overall System Design}

In addition to the above three key technologies, but also need to determine the high-definition image acquisition module and the underlying operating system to determine the overall system framework. First, the IMX178 image sensor supporting 6M, 5M, 1080p and 720p pixels is selected as the source of high-definition images to meet the requirements. Secondly, because the Linux operating system has the advantages of open source code and good network support, this topic will be used as the bottom operation system. According to the research content of the subject and the identified key technologies, the whole framework of the system is obtained. First, around the Hi3516A SOC design hardware, to support the IMX178 sensor module LVDS camera interface, Gigabit Ethernet, high-capacity SD card interface and asynchronous serial communication (referred to as UART) interface; Secondly, the Linux operating system for the underlying software, H .265 for the compression coding standard, Live555 for streaming media transmission base, design software to achieve high-definition video capture, H.265 encoding and real-time streaming media transmission; Finally, according to the project requirements to increase the video file local storage and terminal control functions.

\section{Conclusion}

In the developing process of video surveillance, the storage space is limited and the network bandwidth is limited have become the bottleneck in the process of high-definition, intelligent and network development. This paper promotes the solution to solve this problem. In addition, based on Hi3516A and H265 stream real-time transmission system design, we hope promote the technology continues to mature and get system-level applications.

\section{References}

[1] Jia Xinzhang, Li Jingyuan. Technology Information, Vol. 6 (2014) No 53, p.25-26

[2] Peng Sue, Wang Yunhui, Wang Qunyong. China Security, Vol. 12 (2015) No 27, p.74-76

[3] Qian Xiyuan, Jing Jianfen, Hou XuSiem. Zhejiang University, Vol. 30 (2014) No 19, p.144-145

[4] Wang Kuailiang. Technology Information, Vol. 29 (2008) No 27, p.21-23

[5] Zhang Gongxu, Sun Jing. China Security, Vol. 8 (2013) No 27, p.57-60 